\title{
Catatonia following abrupt discontinuation of clozapine
}

\author{
Dewasmika Ariyasinghe, Ranil Abeyasinghe
}

\section{Abstract}

Clozapine is the mainstay of therapy for resistant schizophrenia. The compliance of medication in this patient group is poor. Instances of catatonia following abrupt discontinuation of clozapine have been reported. We report a case of a 44-year old woman who developed catatonia on two occasions following abrupt discontinuation of clozapine, and who improved after the reinstatement of clozapine. Although only rarely reported, clinicians should be aware of this phenomenon, in order to facilitate psycho-education, early recognition, avoid unnecessary investigations and minimize inadvertent delay of reinstatement of clozapine therapy.

Key words: clozapine discontinuation, catatonia

SL J Psychiatry 2014; 5(2) 27-28

\section{Introduction}

Clozapine is the only atypical antipsychotic which is effective in alleviating both positive and negative symptoms in schizophrenia. It is the medication of choice in the treatment of resistant schizophrenia. Due to unpleasant side effects such as constipation and weight gain, and poor insight, patients may discontinue clozapine without medical advice, resulting in worsening of psychosis, autonomic instability and diarrhoea. A few cases of catatonia following discontinuation of clozapine have been reported in the literature (1-8).

\section{Case report}

A 44 year old woman with schizophrenia presented to a medical ward with a short history of total refusal of food, muteness, rigidity and alternating agitated and retarded behaviour. She had been prescribed clozapine for the last 10 years and was functioning independently, until the death of her mother three months prior to the index presentation. She had discontinued clozapine recently. In the medical ward she was investigated for meningitis and encephalitis but her blood and cerebrospinal fluid investigations, electroencephalogram (EEG) and computed tomography (CT) of brain did not reveal any abnormalities. She was then transferred to the psychiatry unit and clozapine was reinstated. She improved within a week and was discharged on $400 \mathrm{mg}$ of clozapine daily.

She re-presented a month later with a similar clinical presentation characterized by muteness, negativism, double incontinence, refusal of food or fluid and behaviour that alternated between retardation and severe agitation. This presentation too occurred after the patient stopped clozapine. An EEG and blood investigations were done with unremarkable results. She had to be fed through a nasogastric tube. The patient was reinstated on clozapine, but unlike the previous occasion, this time she failed to show a quick recovery. There were no features suggestive of other comorbidities, such as depression. The patient started to show signs of improvement towards the end of the second week of re-starting clozapine. Features of catatonia improved within 4 weeks but she remained functionally impaired with inability to attend to basic needs independently. She remained in the ward for three months and was discharged to a rehabilitation centre due to poor functional recovery and for monitoring of ongoing medication compliance.

\section{Discussion}

Clozapine has a complex mechanism of action. It antagonizes 5HT2, cholinergic, dopaminergic and histaminergic receptors, resulting in up-regulation of these receptors with long term use. Abrupt discontinuation leads to over activity of these receptors, giving rise to a varied clinical picture: diarrhoea, worsening of psychosis or "rebound psychosis", and autonomic instability characterised by intermittent profuse sweating, fluctuating blood pressure, tachycardia, urinary and faecal incontinence $(1,2,6)$. Catatonia on abrupt discontinuation has been reported but is yet to be recognised as a well established syndrome following sudden cessation of clozapine.

Except in one case report where the patient became catatonic after abrupt discontinuation of clozapine due to agranulocytosis, three months after commencement of treatment (5), most other reports indicate that similar to our patient, most who develop catatonia have been maintained on clozapine for many years.

Literature on the management of catatonia due to abrupt cessation of clozapine has been varied. Electroconvulsive therapy (ECT) has been used with good results in two patients $(5,7)$. But Cerit et al report unsuccessful results even after 11 ECTs (8). Zesiewicz et al reported improvement with cyproheptadine (9). Injectable olanzapine had been successfully used by Shareef et al (3). 
Five case reports have described improvement after reinstatement of clozapine only, and the response was quick, usually within a week. Our patient responded well when she presented the first time after discontinuation, but on the second occasion it took longer for catatonia to improve and even after three months she could not function independently.

It is worth considering whether the term 'clozapine withdrawal symptoms/catatonia' is appropriate, since the 'presence of withdrawal symptoms' is also a criterion in the diagnosis of substance dependence syndromes. The Maudsley guidelines clearly differentiates between withdrawal symptoms and symptoms due to abrupt discontinuation of a medication. It is not uncommon to encounter patients who inquire whether the medication to be prescribed is 'addictive'; and this inappropriate wording may have negative repercussions on clozapine therapy, which is one of the most effective treatment options in resistant schizophrenia.

\section{Conclusion}

Abrupt discontinuation of clozapine therapy resulting in catatonia may be an emerging phenomenon. Cholinergic and serotonergic rebound hyperactivity has been implicated (1). The patients and the care givers should be educated about this possibility before the patient is commenced on clozapine. Though reinstatement of clozapine is shown to alleviate catatonia in most cases, almost all of these patients are likely to undergo extensive investigation prior to identification of the correct diagnosis. Further research is required to identify the incidence, prevalence, neurochemical basis and effective management of this phenomenon. A carefully selected name for the condition is also needed to avoid misinformation of the public.

\section{Declaration of interest}

None declared
Dewasmika Ariyasinghe, Ranil Abeyasinghe Faculty of Medicine, University of Peradeniya, Sri Lanka

Corresponding author: Dewasmika Ariyasinghe

Email: dewasmika.ariyasinghe@gmail.com

\section{References}

1. Yeh AWC, Lee JWY, Cheng TC, Wen JK, Chen WH. Clozapine withdrawal catatonia associated with cholinergic and serotonergic rebound hyperactivity: A case report. Clin Neuropharmacol. 2004; 27(5): 216-8.

2. Wadekar M, Syed S. Clozapine-withdrawal catatonia. Psychosomatics. 2010; 51(4): 355.

3. Shareef N, Pandit B, Srivastava AS, Yadav JS. Clozapine withdrawal catatonia: A case report. 65th Annual National Conference of the Indian Psychiatric Society; 2013 Jan 10-13; Bangalore India.

4. Margetiæ B, Aukst-MargetiæB. Neuroleptic malignant syndrome and clozapine withdrawal at the same time? Prog Neuropsychopharmacol Biol Psychiatry. 2005; 29(1): 145-7.

5. Kumar S, Sur S, Singh A. Catatonia following abrupt stoppage of clozapine. Aust N Z J Psychiatry. 2011; 45(6): 499.

6. Lee JWY, Robertson S. Clozapine withdrawal catatonia and neuroleptic malignant syndrome: A case report. Ann Clin Psychiatry. 1997; 9(3): 165-9.

7. Bastiampillai T, Forooziya F, Dhillon R. Clozapine-withdrawal catatonia. Aust N Z J Psychiatry. 2009; 43(3): 283-4.

8. Cerit C, Tuzun B, Akpinar E, Sahan E. Clozapine withdrawal catatonia refractory to ECT: Acase report. Klinik Psikofarmakoloji Bülteni. 2012; 22(3): 275-7.

9. Zesiewicz TA, Borra S, Hauser RA. Clozapine withdrawal symptoms in a Parkinson's disease patient. Mov Disord. 2002; 17(6): 1365-7. 